\title{
Towards the Harmonisation of UML and SDL
}

\author{
Rüdiger Grammes and Reinhard Gotzhein \\ Department of Computer Science \\ University of Kaiserslautern \\ 67653 Kaiserslautern, Germany \\ \{grammes, gotzhein\}@informatik.uni-kl.de
}

\begin{abstract}
UML and SDL are languages for the development of software systems that have different origins, and have evolved separately for many years. Recently, it can be observed that OMG and ITU, the standardisation bodies responsible for UML and SDL, respectively, are making efforts to harmonise these languages. So far, harmonisation takes place mainly on a conceptual level, by extending and aligning the set of language concepts. In this paper, we argue that harmonisation of languages can be approached both from a syntactic and semantic perspective. We show how a common basis can be derived from the analysis of the UML meta-model and the SDL abstract grammar. For this purpose, conceptually sound and well-founded mappings from meta-models to abstract grammars and vice versa are defined and applied. The long term objective is the syntactic and semantic integration of UML and SDL. The key to achieving this objective is a common language core, which can then be extended in different ways to cover further, more specific language concepts, and is sufficiently flexible to support future language add-ins.
\end{abstract}

\section{Introduction}

UML (Unified Modeling Language [1], [2]) is a graphical language for specifying, modelling and documenting software systems with widespread use in industry, standardised by the Object Management Group (OMG). It is a family of notations (e.g., use case diagrams, class diagrams, sequence diagrams, statechart diagrams, deployment diagrams) supporting different views of a system throughout the software life cycle. Recently, the UML 2.0 standard was finalised. The new standard is a major revision of UML 1.x, and introduces, amongst other things, better support for system structure and components.

SDL (System Design Languages [3]) is a graphical specification language for distributed systems and, in particular, communication systems, standardised by the International Telecommunications Union (ITU). It is widely used in telecommunications industry. SDL is a sophisticated set of notations (e.g., MSC-2000, SDL-2000, ASN.1, TTCN), supporting different system views on different levels of abstraction.

With SDL-2000, several important steps towards its future harmonisation with UML were made. For instance, classes and associations including aggregation, composition, and specialisation were added to the language. Furthermore, 
composite states that are similar to submachines in UML statecharts were incorporated. In turn, UML 2.0 introduced structured classes, which extend classes by an internal structure consisting of nested structured classes, ports and connectors. This makes it possible to model architectural aspects of systems in a fashion similar to SDL.

First attempts to harmonise UML and SDL have already been made. The Z.109 standard [4] defines a subset of UML 1.3 [2] that has a mapping to SDL2000. The UML subset is used in combination with SDL, with the semantics based on SDL-2000. In [5], Selic and Rumbaugh define a transformation from SDL-92 to UML 1.3 extended with the Rational Rose real-time profile.

Ultimately, these efforts are directed towards an integration of both languages and the corresponding notations. However, at the time being, UML and SDL still deviate in many ways, making it hard to see whether and when integration might actually be achieved. Differences range from pure syntactic aspects to semantic concepts, resulting from the origin of the languages. Also, it is not clear whether different views of a system even if expressed in notations belonging to the same family are consistent.

A true integration of both languages and the corresponding notations will require a common syntactic and semantic core. This basis may then be extended in different ways, yielding a variety of language profiles. This way, the system developer will be enabled to model different parts of a system using different notations, and to combine them into a single view.

In order to derive a common syntactic and semantic basis, the existing language definitions of UML and SDL should be taken as a starting point. In this paper, we present the results of analysing several corresponding excerpts of UML and SDL, compare them, and derive a common subset. This is done on the syntactical level, by defining conceptually sound and well-founded mappings from meta-models (used to define the abstract syntax of UML, see Section 2) to abstract grammars (used by SDL, see Section 2) and vice versa (Section 3), and by extracting common production rules (Section 4). Results are discussed in Section 5 .

\section{UML Meta-Model and SDL Abstract Grammar}

The definition of a language consists of its syntax and semantics. The concrete syntax of a language includes separators and other constructs needed for parsing the language. The abstract syntax omits these details and contains only the elements relevant for the definition of the semantics. Both the concrete and the abstract syntax of a language can be defined in terms of a grammar, consisting of a set of production rules that define the syntactically correct sentences.

For SDL, a concrete (textual and graphical) syntax and two abstract syntaxes, AS0 and AS1, are defined. The AS0 is obtained from the concrete syntax by omitting details such as separators and lexical rules. Otherwise, is very similar to the concrete syntax of SDL. The abstract syntax AS1 is obtained from the abstract syntax AS0 through a step of transformations followed by a mapping. 
During the transformation, additional concepts are translated into core concepts of SDL as described in the standard.

The abstract syntax of SDL is described in terms of an abstract grammar, similar to BNF. It consists of two kinds of production rules, namely concatenations and synonyms. A concatenation 'lhs ::=(::) rhs' describes the non-terminal lhs (left hand side) as a composite object consisting of the elements denoted by rhs (right hand side). Optional elements are enclosed in square brackets, and alternatives are separated by vertical bars. The suffix '*' describes a possibly empty list of elements, '+' a non-empty list and '-set' a set of distinguishable elements. A synonym 'lhs ::=(=) rhs' describes that the non-terminal lhs is an element of the kind rhs and can not be syntactically distinguished from other elements of this kind.

For the mapping described in Section 3, we assume a normal form of the abstract grammar, where concatenations have no alternatives on the right hand side. The SDL abstract grammar can be easily transformed into this normal form by introducing new synonyms for these alternatives.

Below an excerpt of the AS1, the production rule State-node, is shown. State nodes are composite objects consisting of a state name, a save signalset, and sets of input nodes, spontaneous transitions, continuous signals and connect nodes. Optionally, a state node can have a composite state type identifier (in that case, the state represents a composite state of the respective type).

\section{State-node ::=(::) State-name \\ [On-exception] \\ Save-signalset \\ Input-node-set \\ Spontaneous-transition-set \\ Continuous-signal-set \\ Connect-node-set \\ [Composite-state-type-identifier]}

A meta-model is a model used to define a language for the specification of models. In UML, this meta-model approach is used to define the language syntax. In particular, the abstract syntax of the language is defined using UML class diagrams. This approach is reflective, since class diagrams are UML models, and therefore described in terms of themselves. On top of the model and the metamodel, more layers can exist (meta-meta-models, etc.). UML uses a four layer meta-model structure: user objects (M0), model (M1), meta-model (M2) and meta-meta-model (M3). Every element in a layer is an instance of an element of the direct superordinate layer.

The UML class diagrams used for the description of the abstract syntax comprise packages, classes, attributes, associations and specialisation. Classes in the UML meta-model describe language elements. An occurrence of the language element in the model (M1) is an instance of the meta-model class. Classes in the meta-model can be parameterised by attributes. Attributes describe properties of the language element described by a class. Composition between meta-model classes describes that a language element contains another. General associations 
relate language elements with each other, e.g., a transition with a trigger. The meta-model uses packages, abstract classes and specialisation to structure the abstract syntax.

\section{Defining Mappings Between UML Meta-Model and SDL Abstract Grammar}

In this section, we define precise, conceptually sound mappings from metamodels to abstract grammars, and vice versa. The examples for the reverse mapping can be found in [6]. As it turns out, not every element of the UML meta-model can be mapped. Also, several meta-model elements may have the same representation in the abstract grammar. Therefore, the mapping is not completely reversible. However, it is possible to map every element of an abstract grammar to a meta-model representation. In Section 4, these mappings will be applied to UML and SDL to extract a common syntactical basis.

\subsection{Classes and Enumerations}

$\operatorname{map}(\mathrm{MM}):$ A concrete class of the meta-model represents a language element of the model. E.g., the meta-model class State represents all state descriptions in a UML statemachine. In an abstract grammar, a language element is represented by a specific production rule, namely a concatenation. Therefore, a concrete class in the meta-model is mapped to a concatenation of the abstract grammar. The name of the non-terminal is derived from the class name and the package structure of the meta-model (see below). The right hand side of the concatenation is derived from the class definition (attributes) and context (associations) as defined below (see 3.2, 3.3).

An abstract class of the meta-model describes properties that are common to its subclasses. E.g., the meta-model class Vertex describes properties that are common to states and pseudo-states (initial states, ... ). Since an abstract class can not be instantiated, it does not represent a language element of the model. Therefore, no concatenation is used in the mapping. Instead, we have decided to map an abstract class of the meta-model to another kind of production rule, namely a synonym, of the abstract grammar. In an abstract grammar, a synonym replaces the element on its left hand side with an element of the right hand side. This is a similar to abstract classes in the meta-model, which must be replaced by one of their concrete subclasses in a model. The name of the non-terminal is selected as in the case of a concrete class. The right hand side is derived from the context (specialisation) as described below (see 3.5).

An enumeration in the meta-model is a set of values used to parameterise meta-model classes. E.g., the meta-model class Pseudostate describes different language elements (entry point, exit point, ... ) of the model depending on the value of the attribute 'kind' of the enumeration type PseudostateKind. Enumerations do not directly describe language elements of the model. Therefore, as in the case of abstract classes, no concatenation is used in the mapping. Instead, 


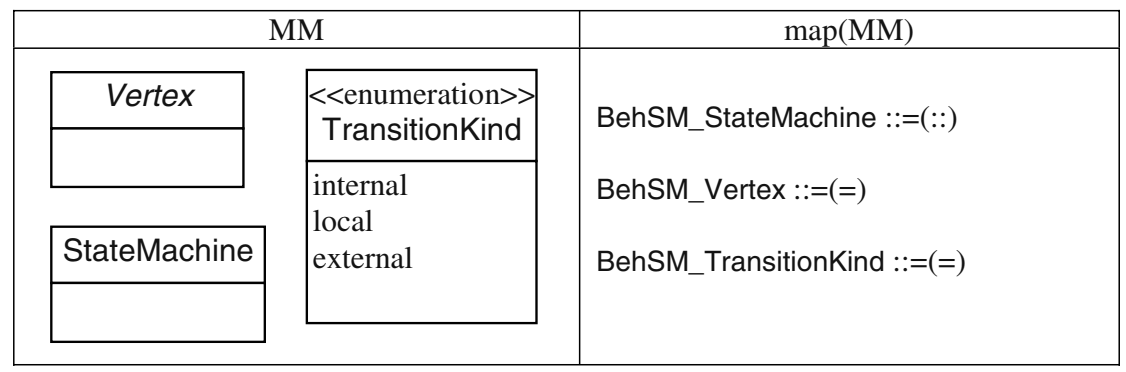

enumerations are also mapped to synonyms of the abstract grammar. This production rule replaces the enumeration by one of its values.

The name of the non-terminals introduced by the mappings described above is the qualified name of the class or enumeration. The qualified name is a sequence of the packages the class or enumeration is contained in (from outermost to innermost) and the name of the class or enumeration, each separated by underscores. E.g., Kernel_Element is the name of the non-terminal introduced by the class Element in the package Kernel. The qualified name is used in order to avoid name clashes between equally named classes in different packages.

Example: The following example comes from the meta-model of UML state machines. It describes two classes, an abstract class Vertex and a concrete class StateMachine. Furthermore, there is an enumeration TransitionKind. All of these elements are contained in the package BehaviorStatemachines (not shown), that we will shortly refer to as BehSM.

StateMachine is a concrete class, and is therefore mapped to a concatenation. The name BehSM_StateMachine comes from the package structure and the name of the class. The abstract class Vertex and the enumeration TransitionKind are mapped to synonyms.

$\operatorname{map}(\mathbf{A G}):$ As mentioned in the mapping from meta-models to abstract grammars, concrete classes and concatenations both represent language elements of the model. Therefore, concatenations of the abstract grammar are mapped to concrete classes in the meta-model. The name of the concrete class is derived from the production rule (see below).

A synonym of the abstract grammar represents a language element that does not appear in the model, but stands for other language elements. E.g., a Datatype-definition in the SDL abstract grammar is a synonym for a Value-datatype-definition, an Object-data-type-definition or an Interface-definition. This is a similar concept to abstract classes in the meta-model, which we have mapped to synonyms in the abstract grammar. However, it is also similar to an enumeration, where the enumeration stands for one of its values. Therefore, we map a synonym in the abstract grammar either to an abstract class or an enumeration. The exact mapping depends on the right hand side of the synonym (see $3.2,3.3$ ). The name 


\begin{tabular}{|c|c|c|}
\hline \multicolumn{2}{|c|}{ MM } & $\operatorname{map}(\mathrm{MM})$ \\
\hline$<<$ enumeration $>>$ & \multirow{4}{*}{$\frac{\text { Transition }}{\text { kind: TransitionKind }}$} & \multirow{4}{*}{$\begin{array}{l}\text { BehSM_TransitionKind }::=(=) \\
\text { INTERNAL } \\
\text { | LOCAL } \\
\text { | EXTERNAL } \\
\text { BehSM_Transition }::=(::) \\
\text { BehSM_TransitionKind /* kind */ }\end{array}$} \\
\hline 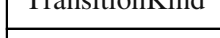 & & \\
\hline $\begin{array}{l}\text { internal } \\
\text { local } \\
\text { external }\end{array}$ & & \\
\hline & & \\
\hline
\end{tabular}

of the class or enumeration is the name of the non-terminal on the left hand side of the production rule.

\subsection{Attributes}

$\operatorname{map}(\mathrm{MM}):$ In the meta-model, attributes of a class represent properties of a language element of the model. E.g., the attribute 'kind' of the meta-model class Transition describes if the transition is internal, local or external. In an abstract syntax tree, an attribute is represented as a sub-node of the non-terminal and corresponds to a class. We have mapped concrete classes to concatenations of the abstract grammar. Therefore, an attribute of a concrete class is mapped to a terminal on the right hand side of the concatenation. We map attributes to terminals since they do not need to be refined any further. The only exception is an enumeration type; in that case we map the attribute to a non-terminal, since we have mapped enumerations to synonyms and non-terminals. The name of the terminal is the name of the type (e.g. Boolean). The name of the non-terminal is derived from the name of the enumeration and the package structure, as defined in 3.1 .

Attributes that are marked as derived carry no additional information and can be omitted. E.g., the attribute 'isComposite' of State can be derived from the number of associated regions. If they are not omitted, additional static conditions are needed to define the dependencies between the original and the derived attributes. Default values of attributes can not be mapped to the abstract grammar. They can be described by static conditions.

Elements of an enumeration represent values of the enumeration type. A value in an abstract grammar is represented by a terminal. Therefore, enumeration elements are mapped to terminals of the abstract grammar. The name of the terminal is the name of the enumeration element. An enumeration is mapped to a synonym of the abstract grammar. Therefore, we map the terminals to the right hand side of the synonym corresponding to the enumeration.

Example: The following example (again from BehaviorStatemachines) contains a concrete class Transition and the enumeration TransitionKind. The classes are mapped as described in the previous section. The attribute 'kind' of Transition is an element on the right hand side of BehSM_Transition. In this special case 
('kind' is an enumeration), it is a non-terminal that refers to the mapping of the enumeration TransitionKind. The name of the attribute is appended as a comment. The enumeration literals of TransitionKind appear as an alternative of terminals on the right hand side of the production rule, written in all caps for better distinction.

$\operatorname{map}(\mathbf{A G})$ : A terminal on the right hand side of a concatenation represents a property of the language element. In the meta-model, an attribute represents a property of a language element. The terminal is therefore mapped to an attribute of the concrete class corresponding to the concatenation. The type of the attribute is the name of the terminal. The name of the terminal can be chosen arbitrarily as long as it does not conflict with other attribute names of the class.

A synonym with only terminals on the right hand side represents an enumeration of values. E.g., the synonym Agent-kind of the SDL abstract grammar is an enumeration of the values SYSTEM, BLOCK and PROCESS. Therefore, the terminals are mapped to enumeration values of the enumeration corresponding to the synonym.

\subsection{Associations}

map(MM): An aggregation or composition between two classes means that one language element contains or is made up of other language elements. E.g., a Region in a statechart contains vertices and transitions. In the same way, a node in an abstract syntax tree can have sub-nodes. E.g., a State-transition-graph of the SDL abstract grammar has a set of State-nodes as sub-nodes. Therefore, we map aggregation and composition to the abstract grammar so that the definition of the aggregated class is a sub-node of the aggregating class. This is achieved by adding the non-terminal corresponding to the aggregated class on the right hand side of the concatenation corresponding to the aggregating concrete class.

A general association between two classes is an association between language elements, in which the elements play a certain role. E.g., a State is associated with a number of triggers, the triggers playing the role of deferrable triggers. In the SDL abstract grammar, two language elements are associated by identifiers. E.g., an Input-node is associated with a Signal by a Signal-identifier on the right hand side of the concatenation corresponding to the Input-node. Therefore, a directed general association is mapped to an identifier on the right hand side of the concatenation corresponding to the concrete class the association originates from. An undirected general association is split into two directed general associations.

An associations with the union property is the union of the associations that subset it. This is expressed by the property subsets. As in the case of derived attributes, associations with the union property are not mapped to the abstract grammar. 


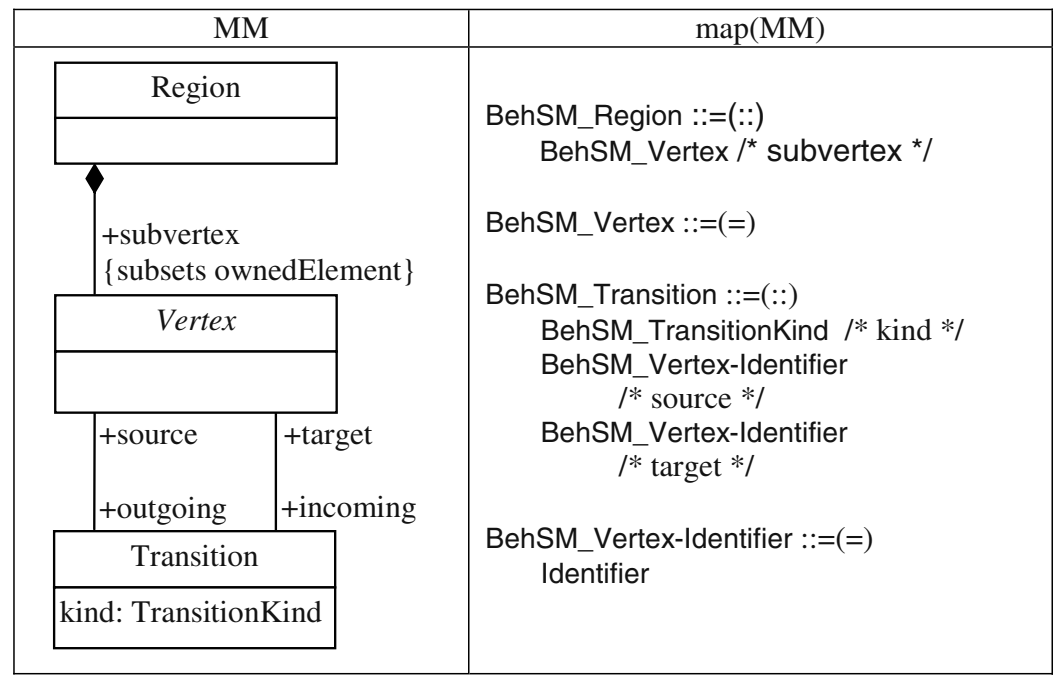

Example: The following example shows the abstract class Vertex and the concrete classes Transition and Region. Region is composed of a Vertex called subvertex. This composition is a subset of the association 'ownedElement' between two Elements. In the AST, BehSM_Region thus has BehSM_Vertex on the right hand side, with the name appended as a comment. Between Vertex and Transition there are two bidirectional associations, which are split into two unidirectional associations respectively. Attributes and associations of an abstract class are not mapped to the corresponding synonym in the AST, since an abstract class is not a synonym for one of its attributes or associations. Instead, they are copied into the respective subclasses, as described in Section 3.5. In this example, Vertex has no subclasses. Therefore, we only have to map the two general associations 'source' and 'target'. To distinguish between general association and composition, an association is mapped to an identifier (in this case, BehSM_Vertex-Identifier) on the right hand side of the corresponding production rule. How the identifier looks like is not further specified. It could be a qualified name like in the case of SDL.

$\operatorname{map}(\mathbf{A G}):$ Non-terminals on the right hand side of a concatenation can stand for an enumeration or a class in the meta-model. In case they represent an enumeration they represent an attribute of the class (see 3.2). In case they represent a class, this class is a sub-node of the class corresponding to the concatenation. This is similar to a class in the meta-model that is composed of other classes. Therefore, in this case we map a non-terminal on the right hand side of a concatenation to a composition in the meta-model. The composing class is the class corresponding to the concatenation; the composed class is the class corresponding to the non-terminal on the right hand side. The role of the classes can be chosen arbitrarily. 
Table 1. Mapping of Multiplicities

\begin{tabular}{|c|c|}
\hline MM & AG \\
\hline $0 . .1$ & [Name] \\
\hline $0 . . \mathrm{n}, 1<\mathrm{n}$ & $\left(\right.$ as $\left.0 . .^{*}\right)$ \\
\hline $0 .{ }^{*}$ & Name * \\
\hline $0 .{ }^{*}\{$ unique $\}$ & Name-set \\
\hline 1 & Name \\
\hline $1 . . \mathrm{n}, 1<\mathrm{n}$ & $(\operatorname{as~1..*)}$ \\
\hline $1 .{ }^{*}$ & Name + \\
\hline $1 .{ }^{*}\{$ unique $\}$ & (as 0..* $\{$ unique $\}$ \\
\hline $\mathrm{n}$ & $\left(\operatorname{as~} 0 . .^{*}\right)$ \\
\hline n...m, $1<\mathrm{n}<\mathrm{m}$ & $\left(\right.$ as $\left.1 . .^{*}\right)$ \\
\hline $\mathrm{n} . .^{*}, 1<\mathrm{n}$ & $($ as 1..*) \\
\hline
\end{tabular}

An identifier on the right hand side of a concatenation identifies a language element that is associated with the language element described by the concatenation. E.g., in the SDL abstract grammar, an Input-node is associated with a Signal by a Signal-identifier. Therefore, we map an identifier on the right hand side of a concatenation to a directed general association in the meta-model. The source of the association is the concrete class corresponding to the concatenation, according to the mapping in 3.1. The target is the concrete class corresponding to the language element referenced by the identifier. The role of the classes can be chosen arbitrarily.

\subsection{Multiplicity}

The following table defines a mapping between multiplicities in the meta-model and the abstract grammar. In UML, multiplicities consist of a lower bound and an optional upper bound, which can be infinite. The property ordered expresses that there is a linear order for the elements. The property unique expresses that no element appears more than once. In the abstract grammar, an optional element is enclosed by square brackets. A possibly empty list of elements is marked by a '*' behind the element, a non empty list by a '+'. A set of distinct elements is marked by the suffix '-set'.

Table 1 shows the mapping of multiplicities between meta-model and abstract grammar. If we use lists in the abstract grammar, the elements are ordered and not necessarily unique. If we use sets, they are not ordered and unique. Therefore, we can only map one of the properties to the abstract grammar. In this case, the property ordered is omitted from the mapping.

\subsection{Specialisation}

In the UML meta-model, abstract classes and specialisation are used frequently to capture common aspects of different classes, and as part of a meta-language 
core reused in several standards (see UML: Infrastructure [1]). For the abstract syntax, abstract classes are not directly interesting, since they can not be instantiated and therefore do not appear in a model, except through their subclasses. Nonetheless we map them to the abstract grammar, to preserve as much of the structure of the meta-model as possible.

$\operatorname{map}(\mathbf{M M})$ : We have to map specialisation to the abstract grammar, and the fact that a specializing class inherits properties of the specialised class. The easiest way to do this is to copy these properties into the specializing classes before the mapping. This has the advantage that redefinition of properties is easy to realise. They are not copied to subclasses that overwrite them.

This is done as follows:

1. For every class that has subclasses, copy all attributes of the class and all associations that originate from this class to each of its direct subclasses.

(a) An attribute is only copied to a subclass if no attribute of the same name already exists, i.e., if the attributed is not redefined.

(b) An association is only copied to a subclass if it is not redefined in the subclass.

2. Repeat step 1 for all subclasses that have new attributes and associations after the last execution of step 1.

In the meta-model, an abstract class can take part in an association. In the model, an instance of a concrete class that specialises the abstract class takes part in the association instead. E.g., a Vertex is associated with Transitions as the source of these transitions. In the model, the source of these transitions is either a State or a Pseudostate. In the abstract grammar, we can express this using a synonym. We have already mapped an abstract class to a non-terminal and a synonym (see 3.1). To map the specialisation to the abstract grammar, we add the non-terminals corresponding to the direct sub-classes of the abstract class to the right hand side of the synonym. This means that every occurrence of the non-terminal (the abstract class) is replaced by a non-terminal (one of the subclasses) in the abstract syntax tree.

To map specialisation to the abstract grammar, we need synonyms. On the other hand, a concrete class can have subclasses, but is mapped to a concatenation (see 3.1). In this case, we transform the meta-model before we perform the mapping. The concrete class with subclasses is replaced by an abstract class of the same name. The concrete class is renamed, e.g. by adding a special prefix, and added as a subclass of the new abstract class. The subclasses of the concrete class are now subclasses of the new abstract class and the mapping can be performed. However, we still have to copy the attributes of the concrete class to its former subclasses, as described above.

Example: The following example is taken from the package Kernel and covers classifiers, classes and associations. Classifier is an abstract class with the 


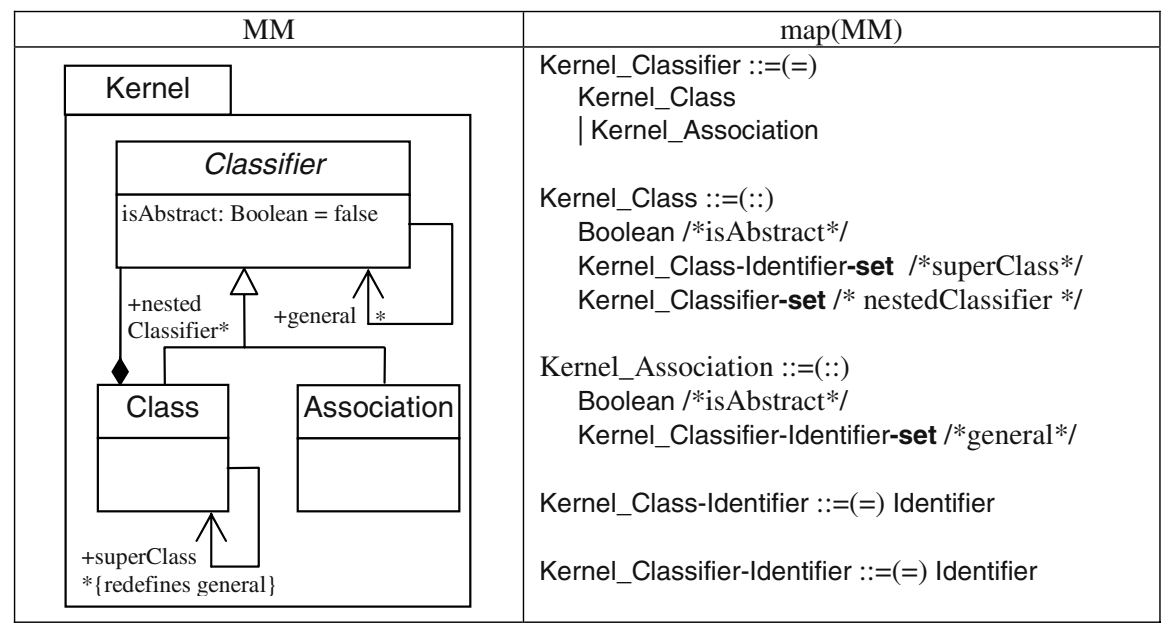

attribute 'isAbstract'. A classifier can be generalised by another classifier, described by the association named 'general'. Concrete subclasses of Classifier are Class and Association. The association 'superClass' between two classes redefines the association 'general'.

Before mapping to the abstract grammar, we have to copy the attributes and associations of the abstract class Classifier to its subclasses. The attribute 'is Abstract' is copied to the classes Class and Association, since no attribute of the same name exists. A new association 'general' from Association to Classifier is added. The association 'superClass' redefines 'general', therefore no new association is added to Class.

The abstract class Classifier is mapped to a synonym. Class and Association are direct subclasses of Classifier; therefore, we add the non-terminals corresponding to these classes on the right hand side of the synonym.

$\operatorname{map}(\mathbf{A G})$ : Non-terminals of the abstract grammar represent language elements. A synonym of the abstract grammar with non-terminals on the right hand side replaces a language element by another. E.g., a Return-node in the abstract grammar of SDL is replaced by an Action-return-node, a Value-return-node or a Named-return-node. We map synonyms to abstract classes in the meta-model. Abstract classes can not be instantiated, but can have instances through their subclasses. Therefore, we map a synonym with non-terminals on the right hand side in the abstract grammar to a specialisation relationship. The specialised class is the class corresponding to the non-terminal on the left hand side. The specialising classes are the classes corresponding to the non-terminals on the right hand side. 


\subsection{Meta-Model Approach vs. Abstract Grammar Approach}

From the discussion so far, it is quite obvious that the meta-model approach to defining an abstract syntax is more expressive than the (context free) grammar approach. As a consequence, the mapping from the SDL abstract grammar to a meta-model is completely reversible. However, this is not the case for the mapping from the UML meta-model to an abstract grammar. Several elements of the UML meta-model can not be expressed in the abstract grammar. They are described in [6]. In consequence, the meta-model approach seems to be preferable as a basis for the harmonisation of UML and SDL. It covers and extends the expressiveness of abstract grammars, and thus seems to be the right choice. However, when it comes to implementing a language by providing tool support, an abstract grammar is still needed. With the mapping defined above, such an abstract grammar can be systematically derived.

\section{Extracting a Common Abstract Syntax from SDL and UML}

Translating the meta-model of UML 2.0 into an abstract grammar supports the comparison of the abstract syntax of UML 2.0 and SDL-2000. In particular, it enables us to examine how the common constructs of SDL and UML are reflected in common parts of the abstract syntax of both languages, and to extract a common abstract grammar.

As it has turned out, some information of the meta-model is lost when it is mapped to an abstract grammar (see Section 3.6). However, the information lost is not important for the extraction, because it is not present in the abstract syntax of SDL.

Instead of mapping the UML meta-model to an abstract grammar, we could apply the mapping from the SDL abstract grammar to a meta-model. This way, no information would be lost, as the meta-model is more expressive. However, the extraction process would not benefit from this choice. Even worse, the extraction would be harder, since the UML meta-model defines a large number of abstract classes with attributes and associations, which would not show up in the SDL meta-model. It would be necessary to either copy the attributes of abstract classes to their subclasses in the UML meta-model (as described in Section 3.5), or to identify common attributes and associations, and shift them to superclasses in the SDL meta-model.

To relate language elements of SDL-2000 and UML 2.0 on a syntactical level, substantial knowledge of both languages is required. In particular, it is necessary to take the semantics of language elements into account. E.g., we need knowledge of the semantics of the language elements to relate the Package-name of a Package-definition in the abstract syntax of SDL with the String of a structured class in the abstract syntax of UML. Also, it can be expected that for some of the common constructs the abstract syntax will be different, although the semantic is the same. In some cases, there might even be a common abstract syntax, although the semantics is different. 
To extract the common abstract syntax of the two languages, we take the production rules for language elements that are similar in UML and SDL, e.g. packages, as a starting point, and compare their right hand sides. For corresponding elements in both sets of production rules that represent similar concepts, the production rules for these elements are compared. If they overlap, we can relate the two elements with each other and include them in the common abstract syntax. We start the extraction with very high level language elements, namely packages and agents/classes, before moving to language elements with a finer granularity.

\subsection{Packages}

Both SDL and UML have a concept of packages for grouping and reuse of elements of the specification. Both support the nesting of packages (2). The abstract syntax of UML describes the contents of a package as a set of PackageableElements, a synonym for all elements that can be contained in a package. SDL describes sets of the elements that can be contained in a package, e.g. Signal-definition-set. Common packageable elements in SDL and UML are agents/classes (3), signals (4) and composite states/statemachines (5).

\begin{tabular}{|l|l|l|}
\hline SDL-2000 (AS1) & Common AS & UML 2.0 (derived AS) \\
\hline Package-definition ::=(::) & Package-definition ::=(::) & Kernel_Package ::=(::) \\
1 Package-name & 1 Package-name & 1 [String] \\
2 Package-definition-set & 2 Package-definition-set & 2 Kernel_Package-set \\
Data-type-definition-set & 3 Signal-definition-set & Kernel_PackageableElement-set \\
Syntype-definition-set & 4 Agent-type-definition-set & Kernel_PackageMerge-set \\
3 Signal-definition-set & 5 Statemachine-set & Kernel_ElementImport-set \\
Exception-definition-set & & Kernel_PackageImport-set \\
4 Agent-type-definition-set & & Kernel_PackageableElement $::=(=)$ \\
5 Composite-state-type- & & S StructuredClasses_Class \\
definition-set & & 5 BehStateMachines_StateMachine \\
Procedure-definition-set & & 3 Communications_Signal \\
\hline
\end{tabular}

\subsection{Signals}

Signal types exist in SDL and UML to describe communication between agents/ objects. Signals have a name (1) and parameters, which are represented by sorts in SDL and properties in UML. While representing similar concepts, the abstract syntax of sorts and properties are different, therefore signals in the common abstract grammar have no parameters. 


\begin{tabular}{|l|l|l|}
\hline SDL-2000 $($ AS1 $)$ & Common AS & UML 2.0 (derived AS) \\
\hline $\begin{array}{l}\text { Signal-definition }::=(::) \\
1 \text { Signal-name } \\
\text { Sort-reference-identifier * }\end{array}$ & $\begin{array}{l}\text { Signal-definition }::=(::) \\
1 \text { Signal-name }\end{array}$ & $\begin{array}{l}\text { Communications_Signal }::=(::) \\
\text { Kernel_Property * } \\
1 \text { String }] \\
\ldots\end{array}$ \\
\hline
\end{tabular}

\subsection{Agent-type/Class}

UML 2.0 introduces structured classes, which are classes extended with internal structure and ports. Structured classes are semantically and syntactically similar to Agent-types in SDL. Both have an internal structure of properties (respectively agents, 9), connectors (channels, 7) and gates (ports, 6). Both agent-types and structured classes can specialise other agent-types and structured classes (2), however SDL only supports single inheritance while UML supports multiple inheritance. Behaviour is associated with an Agent-type as a State-machinedefinition, which consists of a name and a Composite-state-type-identifier (8). Behaviour is associated with structured classes by a Behavior-Identifier (8). Behaviour in the abstract syntax of UML is a synonym for statemachines and other behaviour models. Statemachines are syntactically similar to compositestate-types in SDL. The abstract syntax of the two languages differs slightly, since UML does not have a State-machine-definition. In the common abstract grammar, we include the State-machine-definition but discard the name associated with it, since it does not exist in UML.

\begin{tabular}{|c|c|c|}
\hline SDL-2000 (AS1) & Common AS & UML 2.0 (derived AS) \\
\hline Agent-type-definition $::=(::)$ & Agent-type-definition $::=(::)$ & StructuredClasses_Class $::=(::)$ \\
\hline 1 Agent-type-name & 1 Agent-type-name & 1 [String] \\
\hline Agent-kind & 2 [Agent-type-identifier] & \\
\hline 2 [ Agent-type-identifier ] & 3 Signal-definition-set & Kernel_Classifier-Identifier-set \\
\hline Agent-formal-parameter * & 4 Agent-type-definition-set & 2 StructClasses_Class-Identifier-set \\
\hline Data-type-definition-set & 5 Statemachine-set & [Kernel_Type] \\
\hline Syntype-definition-set & 9 Agent-definition-set & Kernel_ElementImport-set \\
\hline 3 Signal-definition-set & 6 Port-definition-set & Kernel_PackageImport-set \\
\hline Timer-definition-set & 7 Channel-definition-set & Kernel_Constraint-set \\
\hline Exception-definition-set & 8 [Agent-behaviour] & Kernel_Behavior-set \\
\hline Variable-definition-set & & 8 [Kernel_Behavior-Identifier] \\
\hline 4 Agent-type-definition-set & Agent-behaviour ::=(::) & Boolean /*isActive*/ \\
\hline $\begin{array}{l}5 \text { Composite-state-type- } \\
\text { definition-set }\end{array}$ & 8 Statemachine-identifier & Communications_Reception-set \\
\hline Procedure-definition-set & & 7 CompStruct_Connector-set \\
\hline 9 Agent-definition-set & & 9 IntStruct_Property-set \\
\hline 6 Gate-definition-set & & Kernel_Property * \\
\hline 7 Channel-definition-set & & Kernel_Classifier-set \\
\hline 8 [ State-machine-definition & & Kernel_Operation * \\
\hline $\begin{array}{l}\text { State-machine-definition }::=(::) \\
\text { State-name } \\
8 \text { Composite-state-type- } \\
\text { identifier }\end{array}$ & & $\begin{array}{l}\text { Kernel_Classifier }::=(=) \\
4 \text { StructuredClasses_Class } \\
5 \text { SehStateMachines_StateMachine } \\
3 \text { Communications_Signal }\end{array}$ \\
\hline
\end{tabular}




\subsection{Channel/Connector}

Channels/connectors connect gates/ports. In SDL, a channel has one or two channel-paths. In case of two channel-paths, the channel is bi-directional and the originating gate of the first path is the destination gate of the second path and vice versa. In UML, the connector connects two or more ports. In the common AS, a channel is a set of channel-ends (2), which is a pair of ports (3). No direction is specified.

\begin{tabular}{|c|c|c|}
\hline SDL-2000 (AS1) & Common AS & UML 2.0 (derived AS) \\
\hline Channel-definition $::=(::)$ & Channel-definition $::=(::)$ & IntStruct_Connector $::=(::)$ \\
\hline 1 Channel-name & 1 Channel-name & IntStruct_Connector-Identifier \\
\hline [NODELAY] & 2 Channel-end-set & 2 Ports_ConnectorEnd $* / * 2 . . * * /$ \\
\hline 2 Channel-path-set & & [Kernel_Association-Identifier] \\
\hline & Channel-end $::=(::)$ & $t^{2}$ \\
\hline Channel-path $::=(::)$ & 3 Port-identifier & 1 [String] \\
\hline 3 Originating-gate & 3 Port-identifier & [Kernel_Type] \\
\hline 3 Destination-gate & & Ports_ConnectorEnd $::=(::)$ \\
\hline Signal-identifier-set & & $\begin{array}{l}3 \text { [IntStruct_ConnectableElement- } \\
\text { Identifier] }\end{array}$ \\
\hline
\end{tabular}

\subsection{Gate/Port}

Gates/ports are endpoints for channels/connectors. Gates specify valid signals for both directions, while ports have required and provided interfaces $(2,3)$.

\begin{tabular}{|c|c|c|}
\hline SDL-2000 (AS1) & Common AS & UML 2.0 (derived AS) \\
\hline Gate-definition $::=(::)$ & Port-definition $::=(::)$ & Ports_Port $::=(::)$ \\
\hline 1 Gate-name & 1 Port-name & $\begin{array}{l}3 \text { Interfaces_Interface-Identifier- } \\
\text { set }\end{array}$ \\
\hline 2 In-signal-identifier-set & 2 Signal-identifier-set & $\begin{array}{l}2 \text { Interfaces_Interface-Identifier- } \\
\text { set }\end{array}$ \\
\hline 3 Out-signal-identifier-set & 3 Signal-identifier-set & $\begin{array}{l}\text { Ports_Port-Identifier-set } \\
\text { IntStruct_ConnectorEnd-set } \\
1 \text { [String] }\end{array}$ \\
\hline & & $\ldots$ \\
\hline
\end{tabular}

\subsection{Composite-state-type/Statemachine}

Composite-state-types as well as statemachines have a name (1), a sequence of parameters (3) and an identifier of the composite-state-type/statemachine that they specialise (2), if any. In UML, a statemachine has one or more regions that contain states and transitions. The equivalent in SDL is a Compositestate-graph (one region) or a State-aggregation-graph (two or more regions). 
A Composite-state-graph contains a State-transition-graph which contains the states of the Composite-state-type. A Region in UML maps to a State-transitiongraph in SDL. Both contain the states (5) and transitions of the compositestate-type/statemachine. Multiple regions are not included in the common AS, because of the different syntax and semantics in SDL and UML. The common AS for Statemachines can be found in [6].

\subsection{Agent/Property}

Agents and properties are both instances of a type (2) (agent-type in SDL, structured class in UML). Both specify upper and lower bounds for the number of instances (3). While the lower bound in UML is optional, it is required in SDL.

\begin{tabular}{|c|c|c|}
\hline SDL-2000 (AS1) & Common AS & UML 2.0 (derived AS) \\
\hline Agent-definition $::=(::)$ & Agent-definition $::=(::)$ & IntStruct_Property $::=(::)$ \\
\hline 1 Agent-name & 1 Agent-name & Kernel_AggregationKind \\
\hline Number-of-instances & 2 [Agent-type-identifier] & Kernel_Property* /*subset*/ \\
\hline 2 Agent-type-identifier & Number-of-instances & Kernel_Property*/*refined*/ \\
\hline Number-of-instances $::=(::)$ & Number-of-instances $::=(::)$ & [Kernel_Association-Identifier] \\
\hline 3 Initial-number & 3 [Initial-number] & 1 [String] \\
\hline 3 [Maximum-number] & 3 [Maximum-number] & $\begin{array}{ll}2 & \text { 'Kernel_Type-Identifier] } \\
3 & \text { [Kernel_ValueSpecification] }\end{array}$ \\
\hline Initial-number $::=(=)$ Nat & Initial-number $::=(=)$ Nat & 3 [Kernel_ValueSpecification] \\
\hline $\begin{array}{l}\text { Maximum-number }::=(=) \\
\text { Nat }\end{array}$ & $\begin{array}{l}\text { Maximum-number }::=(=) \\
\text { Nat }\end{array}$ & $3 \ldots$ \\
\hline
\end{tabular}

\subsection{State-node/State}

State-nodes in SDL are similar to states in UML, however the syntax is different. Both have a name (1) and an identifier of the composite-state-type/statemachine that is the submachine of this state (2), if any. States are the source of transitions, but in SDL these transitions are associated with the trigger of the transition (Input-node) and in UML with the state itself. The common AS for States can be found in [6].

\section{Conclusions and Outlook}

With regard to recent language developments, harmonisation and finally integration of languages are becoming urgent topics. With more notations being used during the development of a given system, the question whether these views are consistent is gaining importance. Also, in the context of large systems, the use of a mix of notations is getting more likely. Standardisation work to harmonise 
UML and SDL is an important effort towards the objective of having a set of languages that can be used together.

In this paper, we have argued that the harmonisation of languages requires a common syntactic and semantic basis. Following this line, we have first defined conceptually sound and well-founded mappings from meta-models - used to define the abstract syntax of UML - to abstract grammars - used by SDL - , and vice versa. By applying these mappings, we have then extracted common production rules, arriving at a common abstract grammar for several language constructs. While the results were encouraging for structural language elements, it turned out that the coverage was below expectations for behavioural constructs. From this experience, we have drawn the conclusion that an extraction on a purely syntactical basis is not sufficient.

In [6], we have therefore compared language elements on a semantic basis. For this comparison, we chose UML statecharts and SDL process graphs, respectively. UML statecharts have a complete semantics with few variation points. Several attempts to formally define the behaviour of statecharts exist, e.g. [7]. The syntactic comparison of UML and SDL revealed that the abstract syntax of statecharts and process graphs is very different. However, there are several language elements in both languages that have a similar notation and represent similar concepts, despite major syntactic differences. The semantic comparison showed that there is indeed potential for the harmonisation of UML and SDL. However, it also revealed that without a common formal basis, the results that can be obtained are of limited value.

We finally conclude that future work should be directed towards a common semantic core for UML and SDL, with the intention of having extensions of this core to cover further, language specific concepts. Both languages are complex and sophisticated, so this will definitely not be a simple task. However, our experience with the definition of the SDL formal semantics [8] has shown that this kind of work provides valuable feedback to the language designers, finally leading to an even better language.

In a related work, Fischer et al [9] describe a way to generate meta-models from BNF grammars and demonstrate their approach for the abstract syntax of SDL-2000. To capitalise on the advantages of meta-models, they introduce abstract concept definitions and transform generated concrete elements to specialisations of abstract elements.

\section{References}

[1] OMG Unified Modelling Language Specification: Version 2.0 (2003) 61, 70

[2] OMG Unified Modelling Language Specification: Version 1.3 (1999) 61, 62

[3] ITU Recommendation Z.100: Specification and Description Language. Geneva (1999) 61

[4] ITU Recommendation Z.109: SDL combined with UML. Geneva (2000) 62

[5] Selic, B., Rumbaugh, J.: Mapping SDL to UML. Rational Software Whitepaper. (1999) 62 
[6] Grammes, R., Gotzhein, R.: Towards the Harmonisation of UML and SDL - Syntactic and Semantic Alignment -. Technical Report 327/03, Technical University of Kaiserslautern (2003) 64, 72, 76, 77

[7] Börger, E., Cavarra, A., Riccobene, E.: Modeling the dynamics of UML State Machines. In Gurevich, Y., Kutter, P., Odersky, M., Thiele, L., eds.: Abstract State Machines. Theory and Applications, Springer-Verlag (2000) pp. 223-241 77

[8] Glässer, U., Gotzhein, R., Prinz, A.: The Formal Semantics of SDL-2000 - Status and Perspectives. Computer Networks 42 (2003) pp. 343-358 77

[9] Fischer, J., Piefel, M., Scheidgen, M.: A Metamodel for SDL-2000 in the Context of Metamodelling ULF. In: SAM'04. (2004) 77 